\title{
Design of a Hybrid Railway Power Conditioner with Co-phase Power Supply System
}

\author{
Pengfei Xu1,a , Qiao Zhang ${ }^{2}$, Jie Yao ${ }^{3}$, Yantao Chang ${ }^{4}$ \\ ${ }^{1}$ School of Automation, Wuhan University of Technology, Wuhan, Hubei, China \\ ${ }^{2}$ School of Automation, Wuhan University of Technology, Wuhan, Hubei, China \\ ${ }^{3}$ Tongfang Co., Ltd, Beijing, China \\ ${ }^{4}$ College of Electrical and Information Engineering, Hunan University, Changsha, Hunan, China
}

\begin{abstract}
With the widespread use and rapid development of electric locomotives, the negative sequence and excessive phase problems of traction networks have become increasingly prominent. A hybrid railway power conditioner system (H-RPC) for co-phase was proposed, And compared with the traditional RPC system. The paper analyzed its topology and compensation principle. The design of its key parameters was given. The analysis results show that H-RPC has better compensation effect than RPC. Finally, the correctness of the H-RPC compensation effect is verified by simulation.
\end{abstract}

\section{Introduce}

With the maturity of China's high-speed rail technology, DC electric locomotives are gradually being replaced by AC electric locomotives. Since the commutation stage of the AC locomotive generally adopts PWM modulation technology, the power factor of the network side is generally higher, However, as the traction capacity and running speed of the locomotive increase significantly, the increasingly serious negative sequence and traction network over-phase problems are accompanied.

In order to comprehensively solve the above problems, we use phase sequence rotation to balance the single-phase asymmetry of the load in the wiring mode, but the existence of the electric phase separation link limits the further development of the railway to high-speed and heavy load [1]. In terms of active device compensation, the use of hybrid active filter for harmonic suppression is an effective measure [2-5], but it cannot effectively compensate for negative sequence currents. Using static var compensator (SVC) can not only provide reactive power compensation, but also can compensate negative sequence current, but its required capacity is larger [6-8]. The railway power conditioner RPC proposed by Japanese scholars first links the two power supply arms into one whole [9-11], The active power can be transferred between the two arms. Although it has excellent compensation performance and stability, the investment cost is high.

Based on the above background, this paper proposes a new hybrid RPC (ie: H-RPC) for co-phase power supply based on an impedance balanced transformer. This paper will first briefly discuss the topology structure and port wiring mode of H-RPC, and then theoretically analyze the negative sequence management principle of
H-RPC. On this basis, the optimal design method of $\mathrm{LC} / \mathrm{L}$ parameters under fluctuating load is given. and finally, verify the effectiveness of the system compensation effect through simulation.

\section{Topology}

The topology of H-RPC is shown in Fig. 1. The $110 \mathrm{kV}$ (or $220 \mathrm{kV}$ ) grid voltage is reduced by traction main transformer and then reduced to $27.5 \mathrm{KV}$ to supply power to the locomotive.

Compared with traditional RPC, H-RPC has thefollowing significant features:

1) The voltage supplied by the H-RPC to the locomotive is the difference between the two side port voltages $U \alpha, U \beta$ of the impedance balance transformer (ie, the ED port voltage in Fig. 1. is $27.5 \mathrm{KV}, U \alpha(\mathrm{EF})=$ $U \beta(\mathrm{DF})=27.5 / \sqrt{2} \mathrm{KV}$ ), neutral point $\mathrm{F}$ is not connected to two converters, At this point, the load currents of the $\alpha, \beta$ phases are equal in magnitude and opposite in direction.

2) The $\beta$ phase of the H-RPC is connected to the power supply arm through the LC coupling branch. The parameters of the H-RPC must be designed considering the load conditions.

The RPC topology and compensation principles have been widely reported in the literature and will not be described here[9-11].

${ }^{*}$ Corresponding author: aPengfei Xu: 782661859@qq.com 


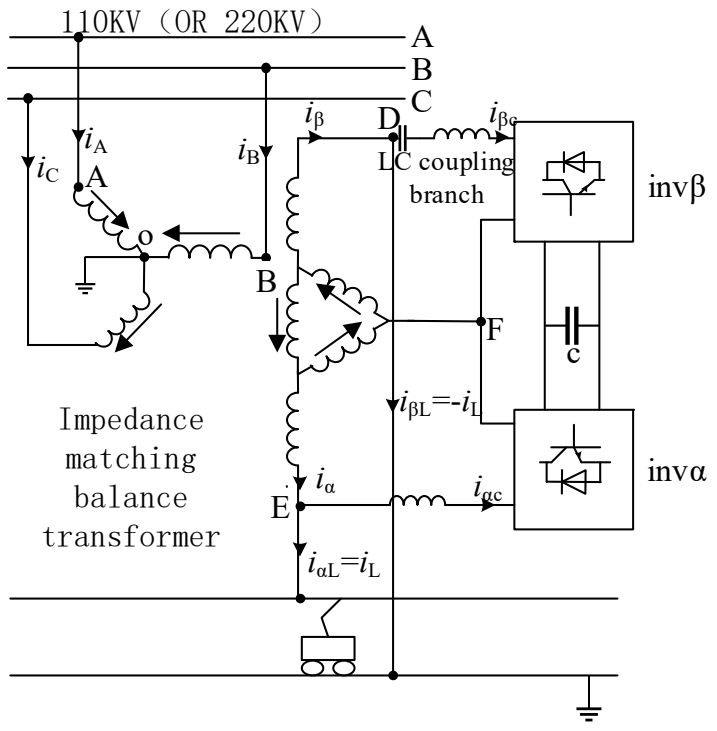

Figure 1. Topology of H-RPC

\section{Compensation principle}

The compensation principle of H-RPC is shown in Fig. 2, The system makes the active components of the two arms equal by transferring the active component of the current $I_{\mathrm{L}}$ in the $\alpha$ and $\beta$ phases, compensate the inductive reactance of the $\alpha$ phase, and compensate the capacitive reactive power of the $\beta$ phase. Finally, the port feeder current is compensated from $I_{\mathrm{L}} 、-I_{\mathrm{L}}$ to $I_{\alpha}^{*} 、 I_{\beta}^{*}$.

There are two things to note: First, due to the power factor angle In the presence of $\theta$, the active current components $I_{\alpha \mathrm{p}}(\mathrm{OA})$ and $I_{\beta \mathrm{p}}(\mathrm{OB})$ of the current $\mathrm{I}_{\mathrm{L}}$ in the $\alpha$ and $\beta$ phases are not equal. $|\Delta I p / 2|=|| I_{\alpha p}|-| I_{\beta p} \mid / 2$ is not equal to 0 most of the time, H-RPC needs to transfer active power most of the time. Second, the feeder port voltage is neither $U_{\alpha}$ nor $U_{\beta}$, but the difference between the two is $U_{\alpha \beta}$.

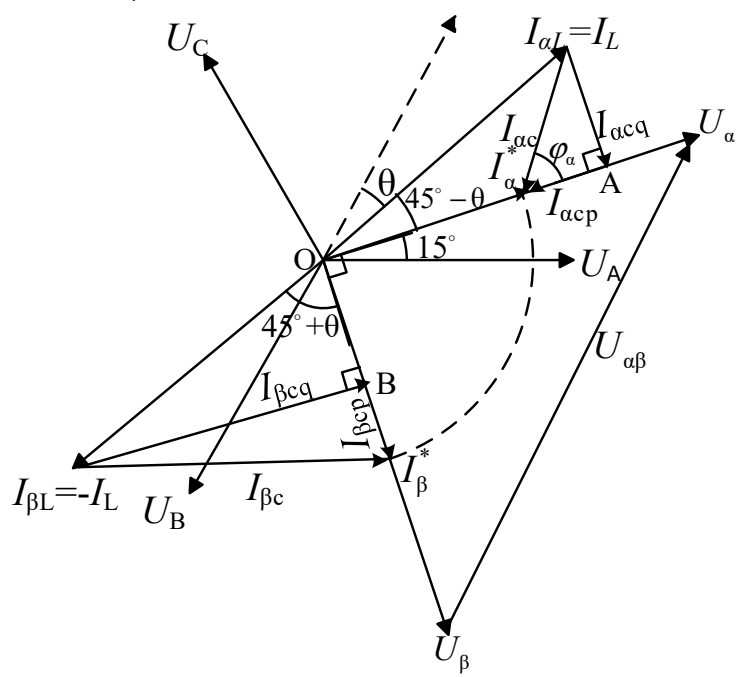

Figure 2. compensation principle

As can be seen from Fig. 2, the relationship between the angle $\varphi_{\alpha}$ (the acute angle between the $\alpha$-phase port voltage $U_{\mathrm{a}}$ and the compensation current $I_{\mathrm{ac}}$ ) and the load power factor $\lambda=\cos \theta$ is

$$
\begin{gathered}
\underset{\alpha}{\varphi_{\alpha}}=\tan ^{-1}\left(\frac{I_{\alpha \mathrm{cq}}}{I_{\alpha \mathrm{cp}}}\right)=\tan ^{-1}\left(\frac{\lambda}{\sqrt{1-\lambda^{2}}}-1\right) \\
I_{\alpha \mathrm{c}}=\sqrt{I_{\alpha \mathrm{cq}}^{2}+I_{\alpha \mathrm{cp}}^{2}}=\varepsilon_{\alpha} I_{L}
\end{gathered}
$$

In the formula

$$
\varepsilon_{\alpha}=\frac{\sqrt{2}}{2} \cdot \sqrt{2-\lambda^{2}-2 \lambda \sqrt{1-\lambda^{2}}}
$$

When $\lambda \in[0.95,1]$, both $\varphi_{\alpha}$ and $\varepsilon_{\alpha}$ increase with $\lambda$

\section{Parameter design}

Since the design of $\alpha$ and $\beta$ phases is similar, Here we only discuss the parameter design of $\alpha$ phase.

\subsection{Design principles}

From Fig. 1 and Fig. 2, it is easy to get the converter port voltage phasor diagram shown in Fig. 3.

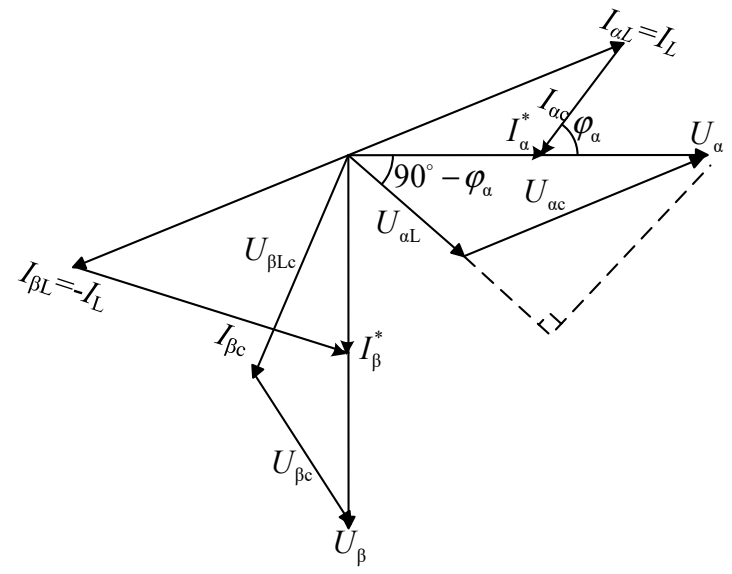

Figure 3. Phasor diagram of outputvoltage.

According to Fig. 3, when $U_{\alpha \mathrm{L}} \perp U_{\alpha \mathrm{cc}}, U_{\alpha \mathrm{c}}$ reaches a minimum, then $U_{\alpha \mathrm{L}}=U_{\alpha} \sin \varphi_{\alpha}$, L coupling reactance $X_{\alpha \mathrm{L}}$ must satisfy:

$$
X_{\alpha L}=\omega L_{\alpha}=\frac{U_{\alpha} \sin \varphi_{\alpha}}{I_{\alpha \mathrm{c}}}
$$

From equations (1) to (4), $X_{\alpha \mathrm{L}}$ is related to load power factor $\lambda$ and load current $I_{\mathrm{L}}$.

the parameter design principle of this paper should meet the minimum voltage of the converter port when the locomotive is heavily loaded (ie, $I_{\mathrm{L}}=I_{\mathrm{L}} \max$ ). For one thing, it can reduce the apparent power of the converter output, reduce costs and losses, and increase economic efficiency. For another thing, it can improve the safety and reliability of the traction system operation.

\subsection{Parameter design}

Fig. 4 shows the port current and voltage phasor diagram of the alpha phase converter. The load current satisfies $I_{\mathrm{Lmin}}=I_{\mathrm{L} 1}=I_{\mathrm{L} 2}<I_{\mathrm{L} 3}<I_{\mathrm{L} 4}=I_{\mathrm{L} 5}=I_{\mathrm{Lmax}}, \quad$ The compensation currents corresponding to the load current are 
respectively $I_{\alpha c 1}, I_{\alpha c 2}, I_{\alpha c 3}, I_{\alpha c 4}$, and $I_{\alpha c 5}$. Among them, $I_{\alpha c 1}$, $I_{\alpha c 3}$, and $I_{\alpha c 4}$ are compensation currents when $\varphi_{\alpha}=\varphi_{\alpha \min }$, representing the boundary conditions of the compensation current. $I_{\alpha \mathrm{cc} 2}$ and $I_{\text {ac5 } 5}$ are the compensation currents when $\varphi_{\alpha}>\varphi_{\alpha \min }$, and represent the general condition of the compensation current. Therefore, it is of universal significance to analyze these five compensation currents. In addition, $\mathrm{OG}, \mathrm{OH}, \mathrm{OF}, \mathrm{OB}$, and $\mathrm{OC}$ in the figure correspond to voltages $U_{\alpha \mathrm{L} 1}, U_{\alpha \mathrm{L} 2}, U_{\alpha \mathrm{L} 3}, U_{\alpha \mathrm{L} 4}$, and $U_{\mathrm{aL} 5}$ on the $\mathrm{L}$ coupling reactance, respectively. $\mathrm{AG}, \mathrm{AH}$, $\mathrm{AF}, \mathrm{AB}$, and $\mathrm{AC}$ correspond to $\alpha$-phase converter port voltages $U_{\alpha c 1}, U_{a c 2}, U_{\alpha c 3}, U_{\alpha c 4}$, and $U_{a c 5}$, respectively.

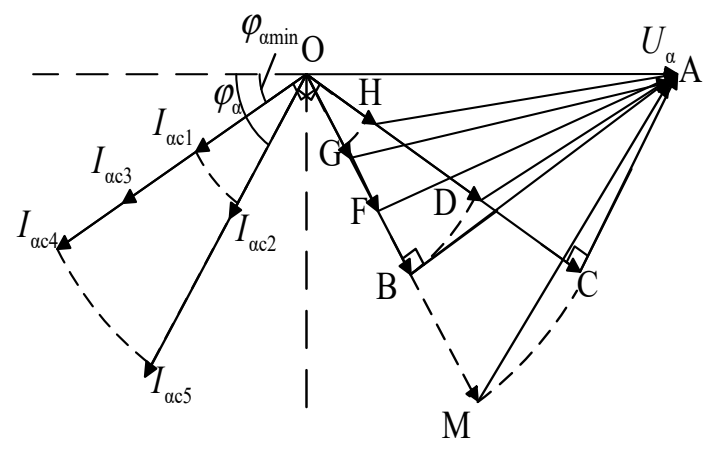

Figure 4. parameters phasor diagram of phase $\alpha$

Since $\varphi_{\alpha}$ increases with $\lambda$, the load current factors corresponding to the compensation currents $I_{\alpha c 1}, I_{\alpha c 3}$, and $I_{\alpha c 4}$ are the lowest in Fig. 4.

According to the parameter design principle mentioned in this paper, $X_{\alpha \mathrm{L}}$ should make the port voltage of the converter lowest when the locomotive is heavily loaded (ie, $I_{\mathrm{L}}=I_{\mathrm{Lmax}}$ ). The load current $I_{\mathrm{Lmax}}$ has two corresponding compensation currents in Fig. 4: $I_{\alpha c 4}$ and $I_{\alpha c 5}$, From the conclusion of Fig. 3, we can see that AB and $\mathrm{AC}$ are the minimum port voltages of converters corresponding to $I_{a c 4}$ and $I_{\alpha c 5}$, respectively. The following two cases are discussed: 1) If the $X_{\alpha \mathrm{L}}$ is determined by the $\mathrm{BO}$ in the $\triangle \mathrm{ABO}$ corresponding to $\mathrm{AB}$, then when the compensation current becomes $\mathrm{I}_{a c 5}$, the port voltage of the converter will become $\mathrm{AD}$, and $\mathrm{AD}<\mathrm{AB}$. Note that when $\varphi \alpha$ increases (ie when $\lambda$ increases), if $X_{\alpha \mathrm{L}}$ is determined using $\mathrm{BO}(\mathrm{ABO})$, the port voltage of the converter will not exceed AB. 2) If $X_{\alpha \mathrm{L}}$ is determined by $\mathrm{CO}(\triangle \mathrm{ACO})$ corresponding to $\mathrm{AC}$, when the compensation current becomes $I_{\alpha c 4}$, the port voltage of the converter will become AM, apparently $\mathrm{AM}>\mathrm{AB}$, a At this time, the output voltage of the converter is too high to be over-compensated, which is not conducive to the work of the converter. To sum up the above two points, $X_{\alpha \mathrm{L}}$ should be determined according to the $\mathrm{BO}$ in the $\triangle \mathrm{ABO}$ corresponding to $\mathrm{AB}$. At this time, Equation (4) can be rewritten as

$$
X_{\alpha \mathrm{L}}=\omega \mathrm{L}_{\alpha}=\frac{U_{\alpha} \sin \varphi_{\alpha \min }}{I_{\alpha c 4}}=\frac{U_{\alpha} \sin \varphi_{\alpha \min }}{I_{\alpha \mathrm{cM}}}
$$

In the equation, $I_{\alpha c \mathrm{M}}$ is the maximum effective value of the compensation current, and satisfies $I_{\alpha \mathrm{cM}}=\varepsilon_{\alpha \max } I_{\mathrm{Lmax}}$ $\varphi_{\text {amin }}$ is the minimum value of the sharp angle between the compensation currents $I_{\alpha c}$ and $U_{\alpha}$.
In order to verify the correctness of the proposed H-RPC, we built a simulation model, And select light-load $(\mathrm{S}=4 \mathrm{MV} \cdot \mathrm{A}, \lambda=0.98)$; medium load $(\mathrm{S}=8 \mathrm{MV} \cdot \mathrm{A}, \lambda=1)$; heavy-load $(S=15 \mathrm{MV} \cdot \mathrm{A}, \lambda=0.95)$ three different loads to simulate the characteristics of the entire locomotive load, The impedance of the coupled branch of the RPC is $19 \Omega$ and is powered by the $\alpha$-phase power supply arm. Other system parameters are shown in Tab. 1.

Table 1. Simulation parameters

\begin{tabular}{lcc}
\hline Parameter & RPC & H-RPC \\
\hline $\mathrm{K}$ & $110: 27.5$ & $110: 19.4$ \\
$L_{\alpha} / \mathrm{mH}$ & 60 & 0.11 \\
$L_{\beta} / \mathrm{mH}$ & 60 & 39.63 \\
$C_{\beta} / \mu \mathrm{F}$ & 0 & 45.8 \\
$C_{\mathrm{dc}} / \mathrm{mF}$ & 20 & 20 \\
$U_{\mathrm{dc}} / \mathrm{KV}$ & 45 & 23 \\
\hline
\end{tabular}

When the load power is $15 \mathrm{MV} \cdot \mathrm{A}$ and the power factor is 0.95 , Fig. 5 and Fig. 6 show the waveforms of the three-phase current of the system network, output current of the secondary side port of the main transformer, voltage and current unbalance of the DC side before and after (0.1s) H-RPC and RPC respectively. we can see that after investing in H-RPC and RPC, The three-phase currents of the system network tend to be symmetrical sine waves, the power factor is close to 1 , and the output currents $i_{\alpha}$ and $i_{\beta}$ of the secondary-side ports are equal in amplitude, with a phase difference of $90^{\circ}$, The power quality has been significantly improved, so both H-RPC and RPC have satisfactory compensation effects under the same load conditions. However, after comparing Fig. 5 and Fig. 6(c) and (d) respectively, we can see that after investing in RPC, The three-phase current unbalance on the network side dropped to $2.1 \%$, the voltage on the DC side stabilized at a given value of $45 \mathrm{KV}$, and after the H-RPC was put into operation, the unbalanced three-phase current on the network side dropped to $0.6 \%$, and the voltage on the DC side was stable at $23 \mathrm{KV}$. This shows that under the premise of accomplishing the same compensation task, The H-RPC has better compensation under heavy load conditions, which verifies the correctness of the parameter design method proposed above.

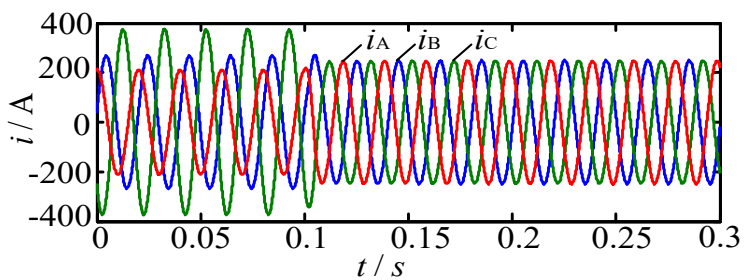

(a) Main transformer primary current

\section{Simulation study}




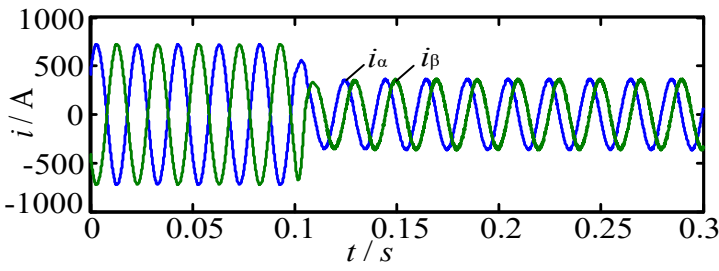

(b) Secondary side port output current of main transformer

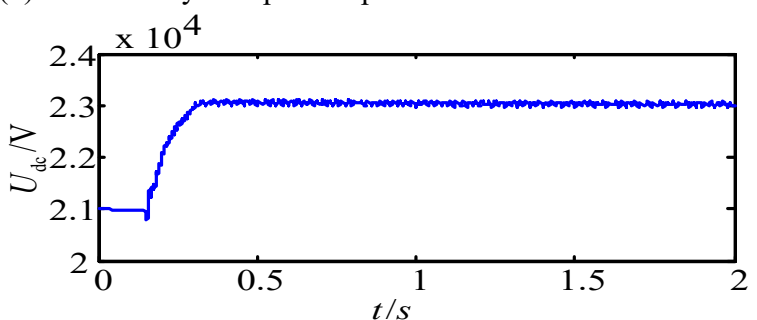

(c) DC voltage

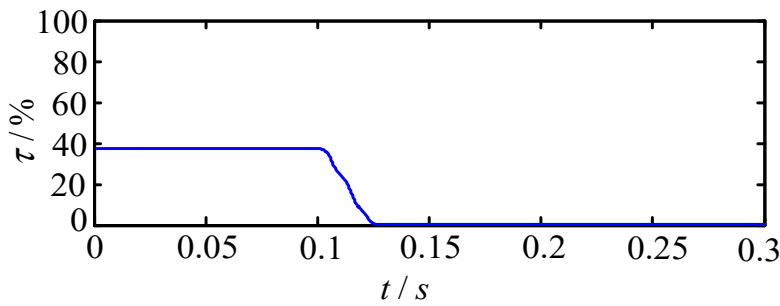

(d) Current imbalance degree

Figure 5. waveforms of system when without or with H-RPC

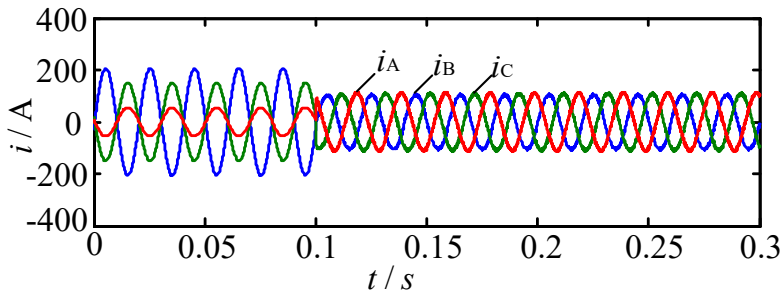

(a) Main transformer primary current

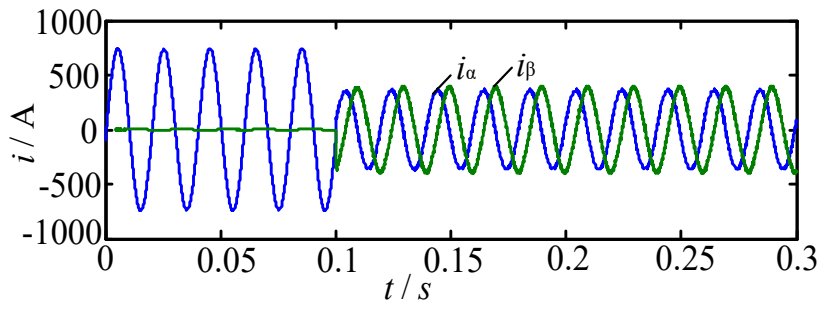

(b) Secondary side port output current of main transforme

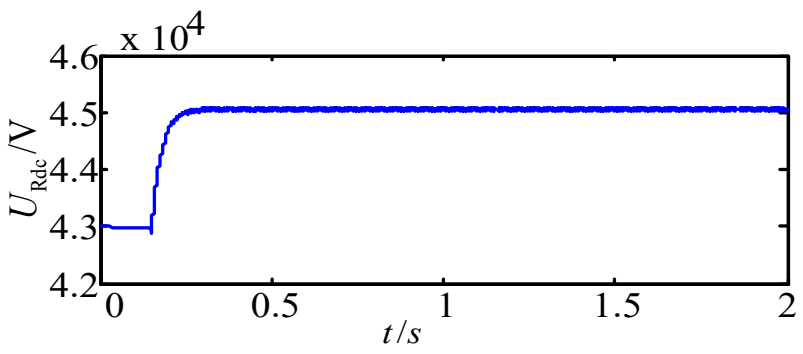

(c) DC voltage

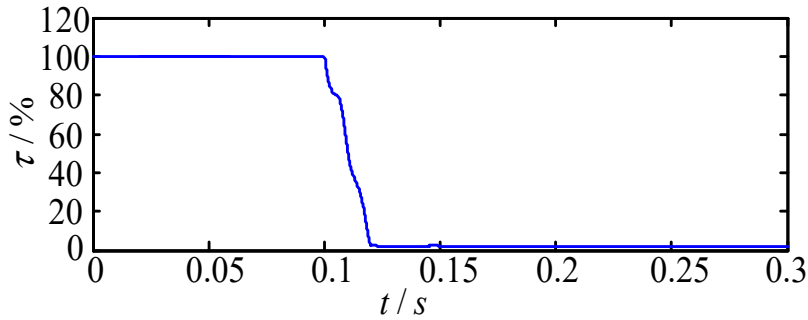

(d) Current imbalance degree

Figure 6. waveforms of system when without or with RPC

\section{Conclusion}

This paper proposes a new system (H-RPC) based on an impedance balanced transformer. The article analyzes in detail the topology of H-RPC and the principle of compensation, and gives the design method of the key parameters of the coupling branch. The system fully exploits the potential of traction main transformer and hybrid compensation branch, which makes H-RPC have better compensation effect than RPC. Finally, the correctness of the system compensation effect is verified by simulation.

\section{References}

1. WU Zhong,WANG Zhi-gang,SONG shu-yong,et al.Simulation research on the influence and management of the electrified railway traction substation on power system [J].Proceedings of the CSEE, 31(S1):77-82 (2011).(In Chiese)

2. WANG Yue, YANG Jun, WANG Zhao-an,et al.Study on hybridpower filter used in electrified railway system[J].Proceedings of the CSEE, (07):23-27 (2003).

3. ZHAO Wei, LUO An,CAO Yi-jia,YU Li. Hybrid var and har-monic dynamic compensator and application to three-two p-hase traction substation[J] proceedings of the CSEE., (28):107-114(2009).

4. ZHAO Wei, TU Chun-ming, LUO An et al. A novel single-phase hybrid active power filter applied to electrical railway[J].proceedings of the CSEE, (21):51-56 (2008).

5. Zhang Dinghua,Gui Weihua,Wang Weian et al. Study and application of a new power quality combined compensation systemfor electrified railway $[\mathrm{J}]$. Transactions of china electrotechnic-al society, (03):189-194 (2009).

6. Zhu Guiping, Chen Jianye and Liu Xiaoyu, "Compensation for the negative-sequence currents of electric railway based on SVC," 2008 3rd IEEE Conference on Industrial Electronics and Applications, Singapore, pp. 1958-1963 (2008).

7. Ding Renjie,Liu Jian,Zhao Yuwei et al. An analysis of instant-aneous power in unbalanced circuits and a method to compensate unbalanced load[J] Transactions of china electrotechnical society, (01):120-124 (2007). 
8. WAN Qingzhu,ZHU Guiping, CHEN jianye et al. Optimum design of compensation capacity for balancing the single-phase traction load[J].Automation of electric power systems,(08):93-97 (2008).

9. WU Chuan-ping, LUO An, XU Xian-yong et al. integrative c-ompensation method of negative phase sequence and harmonicfor high-speed railway traction supply system with $\mathrm{V} / \mathrm{v}$ transformer[J].proceedingsof the CSEE, (16):111-117 (2010).

10. Sijia Hu, Zhiwen Zhang, Yong Li, Longfu Luo, Yijia Cao, Yuehui Chen, Guandong Zhou, Bin $\mathrm{Wu}$, and Christian Rehtanz. A new railway power flow control system coupled with asymmetric double LC branches. IEEE Transactions on Power Electronics, 30(10), pp. 5484-5498 (2015).

11. A. M. Bozorgi, M. Sanatkar Chayjani, R. Mohammad Nejad and M. Monfared, "Improved grid voltage sensorless control strategy for railway power conditioners," in IET Power Electronics, vol. 8, no. 12, pp. 2454-2461, 12 (2015). 